\title{
Civilisations
}

Revue internationale d'anthropologie et de sciences

humaines

58-2 | 2009

Intimités et inimitiés du religieux et du politique en Afrique

\section{Entre contestation et légitimation}

Le religieux en contextes semi-autoritaires en Afrique

\section{Géraldine André et Mathieu Hilgers}

\section{(2) OpenEdition}

\section{Journals}

Édition électronique

URL : http://journals.openedition.org/civilisations/2011

DOI : 10.4000/civilisations. 2011

ISSN : 2032-0442

Éditeur

Institut de sociologie de l'Université Libre de Bruxelles

Édition imprimée

Date de publication : 30 décembre 2009

Pagination : 5-20

ISBN : 2-87263-027-9

ISSN : 0009-8140

Référence électronique

Géraldine André et Mathieu Hilgers, « Entre contestation et légitimation », Civilisations [En ligne], 58-2 | 2009, mis en ligne le 30 décembre 2009, consulté le 30 avril 2019. URL : http:// journals.openedition.org/civilisations/2011 ; DOI : 10.4000/civilisations.2011 


\title{
Entre contestation et légitimation \\ Le religieux en contextes semi-autoritaires en Afrique
}

\author{
Géraldine ANDRÉ \& Mathieu HILgerS
}

\begin{abstract}
$\mathrm{T}$ out au long de l'histoire des sciences sociales, l'analyse des relations qui unissent et désunissent les religions et le politique a donné lieu à un nombre considérable de travaux et a alimenté de multiples débats. L'anthropologie sociale et culturelle consacrée à l'Afrique subsaharienne a souvent analysé ces relations à travers le prisme de l'occulte. Le pouvoir se fonderait sur le contrôle de forces invisibles, sa préservation passerait par un usage secret, habile et efficace de ces éléments-clés dans les stratégies d'accumulation et dans les stratégies de contrôle des ressources. Ces perspectives classiques ont été, en partie, remaniées et reprises pour analyser les configurations contemporaines du religieux et du politique en Afrique (Bernault et Tonda 2000 ; Geschiere 1995 ; Schatzberg 2000, Ellis et Ter Haar 2004 ; Ashfort 2005, et le récent débat autour de la place à faire, ou pas, à « l'occulte » dans la recherche africaniste, Ranger 2007 ; Ellis et Ter Haar 2009 ; Meyer 2009). Ces nouveaux travaux ont notamment souligné que c'est, entres autres, au cœur du religieux que s'élaborent des matrices de représentations et de pratiques collectives qui traversent «l'ensemble du corps social » (Bayart 1993 : 12-13 ; Schatzberg 2000). L'analyse détaillée des relations entre religion et politique semble d'autant plus nécessaire aujourd'hui que, comme l'écrit Bayart, l'Afrique construit "sa propre modernité en dialoguant avec Dieu » (Bayart 1993). Ainsi, ces relations doivent être interprétées en considérant les nouveaux contextes dans lesquels elles se produisent et qui les produisent. L'objectif de ce numéro de Civilisations est de clarifier certains aspects de ces relations en montrant comment les transformations politiques récentes ont permis le renouvellement de pratiques religieuses qui, elles-mêmes, ont alors contribué à (re)façonner le politique'.
\end{abstract}

1. Nous tenons à remercier Joël Noret pour ses remarques et relectures utiles, judicieuses et créatives. 
Les articles réunis ici soulignent chacun à leur manière à quel point l'analyse des relations entre religion et politique ne peut faire l'économie d'une prise en compte des transformations qui ont marqué l'Afrique dans les vingt dernières années. Il convient de rappeler en quelques mots les traits marquants de ces changements décisifs. Après la chute du mur de Berlin, sous la pression des conférences nationales souveraines, des mouvements de contestations populaires, des injonctions des institutions internationales et des bailleurs de fonds, la plupart des pays africains ont connu des aménagements institutionnels. Ceux-ci ont conduit, durant les années 1990, à une décompression des régimes autoritaires et devaient, en principe, mener ces derniers vers la démocratie (Bratton et van de Walle 1997 ; Hyden 2006). Cette libéralisation a eu un impact important sur les mouvements religieux. Elle a contribué au renforcement de certains d'entre eux qui ont profité de la liberté d'association et d'expression pour multiplier leurs groupements, diffuser leur message à travers des organes de presse, et occuper la scène publique afin d'étendre leur influence et d'élargir le nombre de leur fidèles. Des recherches menées dans plusieurs pays ont documenté l'effet de cette libéralisation sur l'expansion des mouvements religieux (notamment Otayek 1999 ; de Surgy 2001 ; Gifford 1998 et 2004 ; Laurent 2003 ; Noret 2004 ; Fourchard, Mary et Otayek 2005 ; Miran 2006 ; Souley et al. 2007 ; Otayek et Soares 2009 ) tandis que des travaux quantitatifs notaient le recul constant des religions traditionnelles ${ }^{2}$.

Pourtant, alors que l'implication croissante dans la sphère publique a été soulignée, les travaux qui ont cherché à analyser de façon systématique l'effet politique des dynamiques religieuses restent peu nombreux et surtout, malgré leur grande qualité, ils demeurent centrés sur des régions précises. Comme le notaient récemment McCauley et Gyimah-Boadi, jusqu'à ce jour, la relation entre religion et démocratie en Afrique sub-saharienne ne semble pas avoir été étudiée de manière suffisamment approfondie (McCauley et Gyimah-Boadi 2009) ${ }^{3}$. Ce numéro n'a bien entendu pas l'ambition de livrer une analyse exhaustive de ce phénomène. Il se présente plutôt comme une contribution qui croise les points de vue et les expériences singulières dans différents pays, afin d'élargir la compréhension de l'articulation entre religion et politique dans les contextes africains contemporains, et de saisir certaines de leurs formes actuelles d'intimités et d'inimités. Pour cela, l'approche choisie ici consiste à étudier ces questions dans des pays à la tête desquels on trouve des régimes semi-autoritaires. Notre hypothèse est que, d'une part, ce type de régimes se multiplient en Afrique et que, d'autre part, l'analyse des mouvements religieux dans ces contextes donne clairement à voir la transformation des modes de perception du politique, la construction de formes d'opposition, et les stratégies de neutralisation de celles-ci par les élites au pouvoir. Avant de poursuivre toutefois, il convient de préciser ce que nous entendons par régimes semi-autoritaires.

2. De tels travaux doivent être considérés avec la plus grande prudence. Il est difficile de chiffrer un tel recul et d'autant que les pratiques ne correspondent pas toujours aux propos tenus aux enquêteurs. Il n'en demeure pas moins que McCauley et Gyimah-Boadi soulignent que dans le round 4 de l'Afro-barometer, enquête réalisée auprès de 26.000 personnes dans 19 pays en 2008, seuls $2 \%$ des interrogés revendiquent la pratique d'une religion traditionnelle (McCauley et Gyimah-Boadi 2009).

3. Notons néanmoins les travaux de Constantin et Coulon (1997), de Gifford (1994) sur l'implication de représentants de l'Eglise catholique dans les processus de démocratisation des pays africains dans les années 1990, ou encore de Haynes (2001) qui montre que l'Eglise catholique et l'Islam minent l'hégémonie des gouvernements autoritaires en élaborant dans les interstices des « dynamiques globales de la démocratisation » des « communautés de croyants » (Haynes $2001: 157)$. 


\section{Contextes semi-autoritaires et implication des mouvements religieux en politique}

Les terminologies multiples utilisées par les politologues contemporains pour décrire les régimes politiques de l'Afrique sub-saharienne aujourd'hui symbolisent bien l'état de la transition entamée dans les années 1990 : " autoritarisme électoral » (Schedler 2006), « démocratie défectueuse » (Collier et Levitsky 1997), " régime hybride », (Diamond 2001 ; Carothers 2002), « nouvel autoritarisme » (Diamond, Lipset et Linz 1995 ; Brooker 2000), « autoritarisme compétitif» (Levitsky et Way 2002), « autocratie libéralisée » (Bratton, Mattes et Gyimah-Boadi 2005). Au-delà des nuances qui distinguent les positions associées à ces terminologies, celles-ci se rejoignent dans la description de régimes qui partagent un même mode opératoire. Tout en affichant un respect des libertés politiques, en utilisant une rhétorique et des institutions formellement démocratiques, ils conservent des traits autoritaires. Sans s'exposer aux risques de la pluralité et de la libre compétition, les gouvernants utilisent les nouvelles institutions pour se maintenir au pouvoir en disposant d'une légitimité qui satisfait les exigences des bailleurs de fonds, et au moins une partie de l'opinion publique. Ces régimes semi-autoritaires, pour s'en tenir à l'appellation générique d'Ottaway (2003), ont été décrits sous l'angle de leur organisation, formelle et informelle. On sait qu'ils autorisent la liberté d'association, le pluralisme politique, que les médias libéralisés y façonnent un espace public et qu'en même temps des dispositifs non officiels de corruption, de népotisme, de violence, ou encore d'intimidation, rendent l'alternance pratiquement impossible. La démocratie et ses élections constituent une façade qui confère au régime sa légitimité sans véritablement l'exposer au risque de la compétition politique.

Ce qu'il importe de documenter plus précisément dans l'analyse de ces régimes, c'est la manière dont ces transformations institutionnelles (nouveaux pouvoirs locaux, élections, liberté d'association et de la presse...) rendent possibles et façonnent un espace imaginaire et pratique au sein duquel s'élabore une critique du pouvoir établi (Hilgers 2009 ; Hilgers et Mazzochetti 2010). Les régimes semi-autoritaires semblent, en effet, traversés par une tension : pour exister ils doivent nourrir et rendre institutionnellement possibles les espérances qu'ils cherchent à neutraliser. En d'autres termes, les aménagements qu'ils concèdent pour préserver leurs légitimités ouvrent de nouveaux espaces qui, même s'ils cherchent à les contrôler, affectent les perceptions et les pratiques du pouvoir. Ainsi, en certains endroits, les transformations institutionnelles semblent dégager des marges de manœuvre susceptibles de renforcer de manière substantielle cette dynamique de démocratisation formelle.

Dans de tels cadres, la capacité à émettre des revendications collectives et à s'immiscer dans le jeu politique est liée à la structure et à l'organisation des collectifs. Avec la libéralisation politique et la progression du processus de décentralisation, les collectifs locaux structurés autour de régimes normatifs spécifiques, nourrissant des intérêts propres et exprimant des revendications, peuvent ainsi participer davantage à une politisation du monde commun et renforcer leur incidence sur les institutions. Il n'est donc pas surprenant que l'importance des mouvements religieux dans les transformations politiques en Afrique ait pu être soulignée. D'abord, dans l'incitation à l'organisation de conférences nationales ${ }^{4}$ (Eboussi Boulaga 1993 ;

4. Toutes les mouvances ne sont pas impliquées de la même manière, les catholiques par exemple ont été beaucoup plus engagés que les pentecôtistes, restés en retrait. 
Gifford 1994 ; Bratton et Van de Waele 1997 ; Banégas 2003), ensuite dans l'engagement parfois critique vis-à-vis des politiques à mettre en œuvre (Bratton, Mattes, Gyimah-Boadi, 2005), enfin par l'investissement de l'espace public, parfois même sous des modalités visant à le délaïciser au point de former un « espace public religieux » (Holder 2009).

Ainsi, si les expériences varient selon l'histoire et les contextes, la littérature est généralement unanime pour souligner l'implication croissante du religieux dans l'univers politique au fil de la dynamique de démocratisation en Afrique subsaharienne. Les trajectoires d'implantation et d'expansion des mouvements pentecôtistes constituent une excellente illustration et ont été abondamment détaillées. Elles s'avèrent relativement analogues dans plusieurs pays d'Afrique à la tête desquels se trouvent aujourd'hui des régimes semiautoritaires : Burkina Faso, Côte d'Ivoire, Gabon, Togo, Nigéria, par exemples.

$\mathrm{Au}$ cours des trois dernières décennies, dans ces pays, les groupes pentecôtistes sont passés d'une position ostentatoirement apolitique à une progressive implication dans la gestion des affaires de la cité. La libéralisation politique, la multiplication et la pluralisation croissante des espaces religieux ont dynamisé l'espace public et ont laissé une plus grande place et une plus grande visibilité à des mouvements dynamiques, soucieux d'élargir le nombre de leurs fidèles et d'exister dans la sphère nationale. Emmenés par de jeunes diplômés souvent sans emplois, les campagnes d'évangélisation, les croisades, les séminaires et les conférences se multiplient. Les prédications stigmatisent avec une vigueur toujours plus grande le désintérêt du politique. Alors que, jusque dans les années 1990, l'implication politique des pentecôtistes s'était limitée au terrain de l'invisible, motivée par la volonté d'assainir un univers traversé par les forces diaboliques, l'objectif désormais ouvertement affiché est aussi de purifier et de spiritualiser le domaine public. La stigmatisation de la corruption, et parfois des inégalités, s'exprime dans des registres symboliques qui prennent une place croissante. Puis progressivement, l'engagement pentecôtiste devient plus concret. L'activisme des responsables religieux devient manifeste. Il s'étend à des projets sociaux, d'éducation, à la lutte contre le sida, le tabagisme, l'alcool, et passe aussi par des organisations non gouvernementales d'obédience pentecôtiste. Cet engagement, ces rencontres, ces discours façonnent un ordre moral et politique. Dans les deux dernières décennies, celuici a pris une ampleur croissante dont la multiplication des travaux scientifiques témoigne et s'est faite l'écho. Le rôle des médias a été extrêmement important dans la diffusion des idéologies pentecôtistes depuis les années 1990. Dans certaines circonstances, ces médias confessionnels ont relayé des problèmes sociaux, des mécontentements ou des grèves dans les secteurs de l'éducation, de la santé, des transports.

L'ampleur et l'efficacité de cette déferlante pentecôtiste et de sa politisation varie selon les régions, selon le nombre de fidèles, selon les « antagonismes religieux et leur degré de conflictualité » (Mayrargue 2004 : 105) ; mais la capacité de ces mouvances à occuper l'espace public est manifeste dans de nombreux pays africains (Corten et Mary 2000), si bien que les hommes forts des régimes en place sont plus ou moins contraints de considérer leur potentiel politique. C'est le cas aussi bien dans des régimes généralement considérés comme davantage démocratiques, le Ghana ou le Bénin dont l'ancien président Mathieu Kérékou s'est converti ${ }^{6}$, que dans des pays aux gouvernements semi-autoritaires. Ainsi, cette

5. Mais aussi dans des pays marqués par des régimes plus démocratiques tels que le Bénin ou le Ghana.

6. Conversion abondamment analysée par Strandsbjerg 2000, 2005. 
mouvance est présente dans l'entourage du président ivoirien Laurent Gbagbo, ou dans celui de politiciens de premier plan au Togo, tel que l'ancien Premier ministre Agbéyomé Kodjo, ou en Zambie autour du président Frederik Chiluba (1991-2002) (Gifford 1998 ; Mayargue 2004 ; Strandsberg 2005). Au Burkina Faso, plusieurs des principaux leaders du mouvement ont rejoint la Commission Electorale Nationale Indépendante (Laurent 2005). Au Gabon, c'est à partir des élections présidentielles de 1998 que le pouvoir prend au sérieux ces Eglises, leur poids sur l'opinion et leur capacité de mobilisation électorale.

Dès ce moment, dans plusieurs pays, la position politique des pentecôtistes devient ambiguë. Le capital social lié aux réseaux pentecôtistes dépasse largement les seuls intérêts religieux et l'implication dans la mouvance a souvent d'autres objectifs que les ambitions spirituelles. De nombreux hommes politiques y adhèrent, la position de convertis occupant des fonctions dans l'administration publique ou dans les entreprises privées « sauve » certains fidèles du chômage, la communauté néo-pentecôtiste constitue un lieu de refuge contre la « désaffiliation sociale » (Castel 1995) ou l'érosion de certains types de solidarités en milieu urbain, mais aussi un réseau au sein duquel les affaires s'organisent. La pentecôtisation de certains espaces politiques explique l'ambivalence de l'État à l'égard de ces Eglises qu'il considère tantôt comme des sectes dangereuses tantôt comme des mouvements potentiellement citoyens, voire utiles pour contrôler les velléités de contestations. Dans quelques cas, les pentecôtistes critiquent plus ou moins vigoureusement le pouvoir en place et les institutions internationales, dans d'autres, les mouvances limitent les mouvements d'opposition en détournant les fidèles des revendications sociales et en proposant à partir de dispositifs de « délivrance » ou de « cure d'âme » des solutions religieuses à l'ensemble des problèmes auxquels le converti peut être confronté (santé, emploi, sorcellerie, etc.).

Cependant, le constat d'une pénétration du pentecôtisme sur la scène politique ne doit pas induire en erreur. Malgré leur dynamisme et leur visibilité, ces mouvements restent largement minoritaires à l'échelle d'une nation. Dans certaines régions, le nombre exact d'adhérents semble difficile à établir, les enjeux politiques sont trop importants pour se fier aux chiffres officiels et les conditions de dénombrement imprécises. En outre, les mouvances sont multiples, les discours politiques ne sont pas unifiés et les affiliations politiques des fidèles sont diverses. Or les variations du champ religieux local et du contexte politique régional et national sont déterminantes dans la forme prise par les mouvements religieux et dans la façon dont ils s'articulent au politique (Mayargue 2008)7. L'occupation croissante de l'espace public par des mouvements religieux ne suppose pas sa pacification, son homogénéisation ou la neutralisation des différences. Au contraire, avec la libéralisation, la segmentarisation des mouvements religieux semble avoir gagné en importance.

\section{La dynamique contemporaine du champ religieux en Afrique : critique et légitimation}

Le constat d'une segmentarisation croissante des mouvements religieux complique les entreprises visant à établir une cartographie claire des relations entre religion et politique. Tout en faisant droit à la complexité de chaque situation locale qu'il étudie, pour dégager une

7. Voir par exemple Mayrague sur la différence entre le Bénin et la Côte d’Ivoire, 2008, p. 15-16. 
perspective générale, nous proposons ici de recourir à une hypothèse inspirée du travail de Pierre Bourdieu.

Etant donné que l'intérêt religieux a pour principe le besoin de légitimation des propositions attachées à un type déterminé de conditions d'existence et de position dans la structure sociale, les fonctions sociales que le religieux remplit pour un groupe ou une classe se différencient nécessairement en fonction de la position que ce groupe ou cette classe occupe dans la structuration des rapports de classe et dans la division du travail religieux. (Bourdieu $1971: 313$ )

On laissera ici en suspend l'idée que la dynamique du champ religieux est entièrement traversée par une axiomatique de l'intérêt, ou qu'elle s'y réduit, pour partir de cette formulation afin de s'interroger sur l'éventuelle existence d'une " homologie entre les positions des producteurs dans la structure du champ et les positions dans la structure des rapports de classe des consommateurs de leurs produits » (Bourdieu 1971 : 319). Ainsi, le lecteur peut trouver dans les articles de ce numéro différents éclairages sur les possibles affinités structurales entre les producteurs d'une idéologie religieuse et leurs consommateurs, ou encore entre des individus qui occupent des positions analogues dans la structure sociale mais dont l'essentiel de l'existence se déroule dans des champs différents.

Il y a une quinzaine d'années, le travail mené par Jean-Pierre Dozon avait déjà mis en lumière de telles affinités en montrant, par exemple, comment certains prophètes contestataires ivoiriens étaient liés à des groupes sociaux dominés (Dozon 1995). De la même manière, d'autres recherches ont souligné que, au Burkina Faso, pendant la colonisation, certains marabouts déambulaient en prêchant la révolte (Hilgers 2009 : 90), alors que les rapports de forces politiques se traduisaient simultanément dans le champ religieux par de nouvelles conversions à la religion de l'envahisseur (Saul et Royer 2001 : 273-276). Le « sous-champ théologique ", pour reprendre la formule bourdieusienne, apparaît ici comme un champ de concurrence dont les idéologies, résultant en partie de cette concurrence, sont prédisposées à être reprises et utilisées pour mener d'autres luttes. C'est ce que souligne Bourdieu lorsqu'il montre que le bas clergé se pose en porte-parole des classes dominées parce qu'il est conduit par sa position dans la structure du champ religieux à émettre une critique dont la formulation est susceptible de faire droit aux représentations et au vécu des groupes, lesquels occupent une position analogue à la sienne dans la structure générale de l'espace social. En effet, la structure des relations objectives entre des instances différenciées dans les rapports de production et de reproduction de biens religieux peut conduire à reproduire de manière transfigurée la structure des rapports de force entre les groupes ou les classes (Bourdieu 1971 : 328). Il reste à établir la validité d'une telle hypothèse dans un contexte plus ou moins libéralisé et marqué par une plus grande segmentation religieuse. Chacun des textes réunis dans ce numéro apporte des éléments de réponse à la question d'une possible correspondance entre la position sociale, la production et la réception d'une idéologie religieuse. L'une des problématiques auxquelles se confrontent à leur manière ces textes est donc de savoir si dans le contexte de la libéralisation, la différenciation croissante de l'espace religieux permet d'établir une relation entre la structure sociale, la position des groupes, la production et la réception des idéologies religieuses.

L'étude de cas de Françoise Bourdarias au Mali cherche à analyser l'affiliation à différents mouvements religieux à Dianguinabougou, « un des quartiers les plus récemment urbanisés de Bankoni », zone urbaine périphérique au Nord de Bamako. Son texte permet d'interroger 
de manière empirique cette hypothèse. En effet, l'affiliation religieuse apparaît ici « comme un remède au désordre et à la transformation des liens familiaux et conjugaux » dans un contexte d'incertitude, d'insécurité économique et de domination. Au-delà d'une religiosité forte qui marque tous les niveaux sociaux, Bourdarias démontre empiriquement la manière dont les pratiques liées au contrôle et à la reproduction ou à la subversion des rapports de domination qui façonnent le politique varient selon la position des groupes dans l'espace social et donc selon leurs ressources matérielles et symboliques. Dans sa perspective, un groupe occupant une position dominante a tendance à produire une idéologie religieuse spécifique qui renforce les appareils du pouvoir et les légitime tandis que les groupes dominés, aménagent, contournent, résistent et tentent de construire des "contre-hégémonies ». Dans la contribution de Bourdarias, le politique est compris comme l'ensemble des principes générant les relations qui régissent les rapports entre les hommes et les rapports que l'homme entretient avec la nature. Il ne se limite donc ni à un lieu ni à un espace. Il est omniprésent mais diffus et nourrit des rapports de force, des oppositions, des conflits ouverts, sourds ou larvés.

Différents rapports entre religion et politique se donnent à voir dans les transformations suscitées par les réformes des années 1990. Les problèmes liés au lotissement que Bourdarias étudie comme une extended case analysis en fournissent un bon exemple. Les politiques publiques d'aménagement de l'espace urbain sont un lieu particulier où s'illustre la manière dont le religieux et le politique interagissent pour les groupes dominés. Les politiques d'aménagement font l'objet de mobilisations locales qui s'appuient sur des mouvements religieux et qui conduisent parfois à des réaménagements des politiques publiques. Généralement entamés sous le couvert de l'idéologie participationniste des institutions internationales, ces lotissements qui accompagnent l'instauration de la décentralisation engendrent souvent une dégradation des conditions de vies, de nouvelles exclusions et des souffrances sociales (Hilgers 2008), et se soldent par une reconfiguration des conditions d'existence et des rapports de domination.

Ce que montre Bourdarias, c'est la manière dont, dans un tel contexte, les populations pauvres sollicitent les mouvements religieux pour obtenir du soutien et faire valoir leurs droits. Lorsqu'elles n'obtiennent pas ou peu de résultats, elles sont contraintes de quitter un espace en voie de gentrification, tandis qu'une nouvelle catégorie d'habitants au profil socioéconomique fort différent s'installe. Ces nouveaux résidents, commerçants, employés d'administrations, techniciens, sont dotés de ressources monétaires, de capital social, de titres scolaires et ont des relations plus aisées avec l'administration. Les habitants pauvres et/ ou expulsés, désormais au centre de relations hiérarchiques marquées, sont confrontés à la réussite de ces nouveaux notables dont les richesses leur rappellent l'importance des réseaux et des voies corruptives dans les dynamiques d'ascension sociale. Cette reconfiguration des conditions d'existence des groupes dominés et des rapports de force explique, selon Bourdarias, le succès populaire de différents mouvements religieux apparus dans les années 1990 dans ce quartier. Ainsi par exemple, pour l'Ançar-Dine, mouvement musulman dont le siège s'est installé à Dianguinabougou en 1991, la réorganisation de la société passe par « l'extension de la sphère domestique » au politique et une exigence de transparence qui inverse les rapports entre gouvernants et gouvernés. L'appareil d'État et l'ordre économique ne sont pas ouvertement critiqués mais l'éthique de vie qu'affichent les fidèles, la rigueur, l'honnêteté, la pureté morale qui sont prêchées les conduisent à juger les pratiques illégales 
et ostentatoires des puissants, à les condamner et à inverser ainsi le stigmate auquel leur pauvreté les condamne. "Cette inversion des regards et de la force d'attraction remet en cause les liens de clientélisme qui conditionnent la réussite sociale en milieu urbain. Ainsi se dessine en creux l'évaluation du monde politique et économique, les voies de sa réforme et le rôle dévolu aux croyants dispersés dans le monde ». Dans les discours, la citoyenneté s'impose ainsi à la fois comme une valeur démocratique et comme la "capacité à maîtriser les rapports sociaux et à les conformer à la tradition religieuse ". Dans ce cas précis, il s'agit pour le mouvement de l'Ançar-Dine de diffuser au-delà du cercle des croyants des règles de vie conformes à « l'intérêt public ».

Cette moralisation de la vie publique par les instances religieuses prend d'autant plus de sens et d'importance que la quête mystique correspond souvent à la recherche d'un nouveau cadre social, de nouveaux rapports de confiance dans un univers marqué par les transformations urbaines et les ruptures dans les formes de solidarités, d'échanges, de travail, de rapports de propriété qu'elles impliquent parfois. Ce sont des mouvements religieux divisés que nous décrit Bourdarias. Au moment où les luttes foncières et le militantisme dirigés contre l'État échouent, certaines mouvances continuent à recruter des fidèles dans les populations les plus précarisées des quartiers périphériques. Les causes du désordre et de l'inégalité sont désignées. Il s'agit avant tout de l'État et de ses élites. Ces mouvements religieux populaires cherchent à reconfigurer les relations sociales et politiques locales en partant des principes d'une morale religieuse qui apparaît elle aussi comme l'expression particulière d'intérêts politiques.

L'article d'Abdoulaye Sounaye fait directement écho à l'analyse de Françoise Bourdarias. Au Niger, le processus de démocratisation entrepris au début des années 1990 a également contribué à l'émergence de nombreuses organisations de la société civile, parmi lesquelles des organisations islamiques qui sont parfois apparues comme une force d'opposition visant à redéfinir les normes de conduite de la vie publique. Ces organisations islamiques s'adressent aux exclus, victimes des pratiques délétères des dirigeants politiques, et fournissent l'occasion aux leaders religieux qui les soutiennent et s'y impliquent de revaloriser leur position mise à mal par la vague de démocratisation. Ces leaders religieux critiquent l'élite politique qui, formée dans des systèmes éducatifs occidentaux et/ou occidentalisés, n'a pas l'Islam comme modus operandi de l'action politique. À travers le prisme d'une culture islamique renouvelée et perçue comme radicale par l'Occident, ces élites islamiques revendiquent une participation, par la médiation de leur autorité religieuse, à une sphère politique de laquelle ils seraient exclus par la sécularisation. En réclamant une purification religieuse des pratiques politiques, ces leaders musulmans soutiennent la population, écartée des bénéfices du pouvoir. Pour autant, cette radicalisation religieuse ne prend pas la forme d'un mouvement social à part entière. En effet, la démocratisation a entraîné un affaiblissement du pouvoir des leaders religieux et engendré des oppositions plus nombreuses entre les groupes musulmans eux-mêmes. Certains groupes radicaux cherchent donc à recouvrer un prestige diminué par ce qui leur apparaît comme l'exclusion de la religion de la sphère publique. Dans ce cas, la position de ces leaders dans l'espace social les conduit à être les porte-paroles des dominés. Cependant, la socialisation de ces leaders religieux, alphabétisés et instruits, les conduit également à investir dans des domaines tels que l'éducation ou les médias (forums de discussion radio, pamphlets) qui touchent aussi les jeunes intellectuels, les élites. On peut donc faire l'hypothèse que le déplacement de la position des leaders religieux dans l'espace 
social n'a pas pour autant suspendu les affinités d'habitus qu'ils pouvaient avoir avec d'autres élites. En effet, armés de la conviction que la réislamisation de la sphère politique passera par l'adhésion de celles-ci, les leaders religieux cherchent également à convertir les jeunes intellectuels, futurs hommes au pouvoir.

Un tel activisme politique demeurait impossible à mettre en œuvre sous les régimes antérieurs. Mais la libéralisation politique a facilité le développement de différentes organisations islamiques. L'intention initiale de certaines d'entre elles était de créer un parti politique islamique. Cependant, en raison de la laïcité de l'État, cela ne fut pas possible. Elles se sont donc constituées en associations afin de se mobiliser contre les politiques sociales qui selon elles mettaient à mal la pérennité de l'identité religieuse des musulmans.

Ce que montre le travail de Sounaye, c'est la tension entre l'État et les groupes musulmans accusés de vouloir islamiser la vie publique. Malgré un cadre constitutionnel d'inspiration française, à la suite de la vague de démocratisation, le maintien d'un régime de laïcité a été compliqué par l'intensification des relations entre les sphères religieuse et politique. Ces relations ont conduit à adoucir la sécularisation du cadre politique et à laisser une plus grande place aux organisations religieuses, à leurs initiatives et à leurs contestations. $\mathrm{Au}$ final, l'activisme social et politique des musulmans engagés donne aujourd'hui lieu à la construction d' " un sécularisme ambigu ». L'obligation de prendre en compte les dimensions religieuses pour asseoir une légitimité politique n'a pas permis l'imposition d'une démocratisation pleinement laïque. Au contraire, la dynamique de démocratisation a paradoxalement permis à certains chefs musulmans de formuler des objections contre le processus de démocratisation au nom de la sécularisation qu'elle implique et qui soulève des questions sur le statut de l'Islam, religion de la majorité des Nigériens, et sur son rôle dans la sphère publique. On le voit, ici, le processus de libéralisation politique et la laïcisation de la gestion des affaires publiques a placé au centre du débat deux questions importantes : celle de l'identité religieuse du pays et celle de la représentation de cette identité par les institutions de l'État et le pouvoir politique. Si de telles questions et les débats qu'elles impliquent ne se posent pas uniquement dans un contexte marqué par une démocratisation progressive, selon l'auteur, elles deviennent inévitables avec les élections « comme principal mode de dévolution du pouvoir ». Ainsi, la démocratisation a facilité l'émergence de ces discours tout en leur donnant un nouveau souffle. Des leaders musulmans s'expriment dans l'espace public et s'opposent au principe d'une constitution qui sépare la religion du politique. Selon eux, la séparation de l'État et de l'Islam aurait conduit à la faillite morale de l'État. Ici, pour reprendre une formule que Gauchet utilise dans un autre contexte et dans un autre dessein, la démocratie fonctionne contre elle-même. Elle permet l'émergence d'organisations qui vont la critiquer parce qu'elle tient à distance l'autorité des chefs religieux. Sans leur participation, elle ne peut, selon eux, qu'échouer dans sa mission de bonne conduite des affaires publiques. Pour Sounaye, l'objectif n'est pas tant de savoir si l'attachement à l'Islam induit un rapport spécifique à la démocratie (Bratton 2003) mais plutôt d'établir en quoi la transformation du champ politique a induit une transformation du religieux et de la participation des adeptes dans l'espace public.

Les processus de démocratisation n'ont pas seulement fait émerger des organisations de musulmans qui ont cherché à faire entendre leur voix au niveau politique, elles ont aussi stimulé différentes initiatives socioculturelles visant à construire une économie morale qui s'étend au-delà de la sphère politique, transforment la sphère islamique et redéfinissent les 
contours de la société civile. Les communautés de prière et la formation (l'enseignement) incarnent très concrètement le discours anti-séculariste. On y encourage l'affiliation à des partis politiques et à des associations de la société civile pour faciliter une « réislamisation » de la société et la pénétration des principes musulmans dans la gestion des affaires publiques. Parallèlement aux discours et aux initiatives de "réislamisation », l'usage de symboles islamiques dans les rituels d'État se multiplie, signe de l'obligation qu'a l'élite politique de prendre en considération les exigences religieuses. Dans un contexte semi-autoritaire où le pouvoir cherche à dominer et contrôler toute forme d'autorité, la maîtrise des autorités religieuses s'avère nécessaire. Toutefois, le gouvernement doit aujourd'hui trouver un équilibre, entre sa dépendance aux aides étrangères qui le contraint à maintenir une couverture laïque, en même temps que sa quête de légitimité sur le plan national le contraint à faire droit aux mouvements islamiques. La démocratisation a conduit à l'affirmation des identités religieuses dans l'espace public. De nombreux musulmans attendent désormais que leurs leaders politiques partagent et affirment leur conviction religieuse.

Lorsque les mouvements religieux sont plus marginaux, la situation diffère. Dans son article, Anne Mélice décrit en détail l'attitude ambiguë de l'Église kimbanguiste dans ses rapports au pouvoir. Derrière un apolitisme de principe, se tissent des relations étroites avec les gouvernements successifs du Zaïre puis du Congo. L'attribution d'un fondement divin à toute forme et à tout exercice du pouvoir temporel place les adhérents kimbanguistes dans une position liminaire. Officiellement, le mouvement se tient à l'écart des rapports de force politiques qui régissent le pays, mais dans les faits les élites kimbaguistes font preuve d'une malléabilité idéologique et d'un opportunisme politique qui les conduisent à légitimer toute transformation qui s'opère au sommet de l'État.

A l'inverse de ce qu'on a vu avec les mouvements islamiques radicaux au Niger, dans la République Démocratique du Congo, depuis la période mobutiste, ce « kimbanguisme officiel » ressemble à s'y méprendre aux théologies africaines décrites par Mbembe, celles qui ont déculpabilisé le pouvoir postcolonial, exonéré la " délinquance d'État » et légitimé les principes autoritaires (1988 : 193). Ici « les topologies cosmologiques sont toujours des topologies politiques naturalisées » (Bourdieu $1971: 330$ ), elles jouent un rôle de légitimation et de justification du pouvoir quel qu'il soit. Néanmoins, selon Mélice, ce kimbanguisme officiel, progressivement érigé en mode de légitimation du pouvoir de l'État, doit être distingué du « kimbanguisme des Kimbanguistes » inscrit dans le prolongement des origines du mouvement et marqué par une position plus critique à l'égard du pouvoir. Au-delà des versions officielles, il existerait donc une théologie critique. Cependant, dans le contexte politique difficile de la RDC, la condition du maintien et de la croissance d'un mouvement semble être déterminée par sa capacité à soutenir le pouvoir, fût-ce de manière implicite. Les autorités voient d'un bon œil le travail spirituel de légitimation même si le fondement divin que le mouvement attribue au pouvoir se situe à mille lieux des discours officiels sur la démocratie et la bonne gouvernance. Les grands prophètes du mouvement éclairent ainsi les effets de la providence sur la destinée politique de la RDC et de Joseph Kabila. C'est pourquoi le kimbanguisme officiel est le seul kimbanguisme à occuper l'espace public. Ici encore, on voit bien que « le maintien de l'ordre symbolique contribue directement au maintien de l'ordre politique tandis que la subversion symbolique de l'ordre symbolique ne peut affecter l'ordre politique que lorsqu'elle accompagne une subversion politique de cet ordre » (Bourdieu 1971 : 328), c'est-à-dire lorsqu'émerge une critique explicite du pouvoir 
politique. Il reste donc à établir dans quelle mesure cette distinction entre kimbanguisme officiel et " kimbanguisme des Kimbanguistes » traduit ou non une homologie structurale entre champ religieux et espace social.

Dans des contextes semi-autoritaires où l'insoumission n'est pas sans risque, l'espace religieux constitue un interstice privilégié où s'exprime la contestation politique. Le texte de Vincent Dississa analyse les usages détournés de chansons populaires qui scandent les funérailles congolaises ( "matanga »). Il illustre la manière dont ces funérailles constituent des moments révélateurs où s'expriment la rancœur, la crainte et la lassitude du pouvoir de l'État. Dississa décrit les funérailles congolaises et leur évolution en montrant ce qu'elles doivent aux transformations des contextes politiques au sein desquels elles se déploient. La restriction des libertés qui caractérise le Congo-Brazzaville depuis un peu plus d'une dizaine d'années, façonne les matanga et les érige en un espace informel de contestation. Selon l'auteur, les chansons populaires se dotent dès lors une fonction neuve : la dénonciation. Dénonciation des abus, des manquements et de l'opportunisme des hommes au pouvoir ; énonciation des errements, de l'abandon et de la marginalisation d'une large frange de la population. Il est significatif que les principaux acteurs de cet usage politique des funérailles soient des jeunes au chômage, et plus largement désoeuvrés, délaissés par les pouvoirs politiques après avoir parfois été instrumentalisés comme miliciens dans les guerres civiles. Ici, les funérailles constituent un espace de subversion, de critique, de dénonciation des abus politiques où les plus démunis peuvent faire entendre leurs voix.

Ces situations diverses soulignent que si les croyances religieuses et spirituelles renvoient à des conceptions du monde qui sont toujours déjà politiques, ce que les acteurs font concrètement de ces conceptions est profondément affecté par la contingence des évènements et par les nouvelles configurations sociales. Les transformations qui ont marqué l'Afrique subsaharienne au cours des vingt dernières années et que l'on a rapidement évoquées ci-dessus ont fourni des cadres permettant de renforcer la portée des contestations. Les changements institutionnels ont donné l'occasion aux groupes disposant d'une organisation efficace et de réseaux de valoriser leur conception de la vie bonne. Loin de pacifier le jeu politique, cette politisation du monde et cette démocratisation plus ou moins grande de la sphère publique a pu conduire à des conflits, voire à des situations paradoxales dans lesquelles l'ouverture démocratique a permis à des mouvements radicaux de revendiquer une théologisation du politique.

Pour établir l'importance et clarifier le rôle des modes de participation religieux dans le domaine politique, ce numéro de Civilisations privilégie largement une analyse centrée sur la relation entre les mouvements religieux et l'État. L'option prise a été de documenter cette relation à travers des études de cas qui permettent de saisir précisément l'interaction entre religion et politique telle qu'elle se décline concrètement tout en mettant en lumière les mécanismes généraux qui sous-tendent cette relation dans des contextes politiques caractérisés par leur hybridité. En effet, on l'a dit, les nouveaux contextes politiques qui ont émergé suite à la libéralisation des régimes autoritaires dans les années 1990 se sont avérés propices à une implication croissante des mouvements religieux dans l'espace public et politique. Pour autant, comme l'indiquent les différentes contributions de ce numéro, le rôle des mouvements religieux dans l'espace politique n'est pas univoque. Qu'ils s'opposent ou qu'ils légitiment le pouvoir, ils fournissent toujours des supports et des repères symboliques pour mettre en forme et interpréter le monde social. Tantôt ils justifient le pouvoir en place, 
tantôt ils formulent des critiques sociales et politiques, et revendiquent une reconnaissance. L'analyse des relations entre religion et politique et plus précisément entre religion et démocratisation est loin d'être épuisée. Modestement, ce numéro constitue une contribution à cette entreprise collective.

\section{Références citées}

BAnEgas, Richard, 2003. La démocratie à pas de caméléon. Transition et imaginaires politiques au Bénin. Paris : Karthala et CERI.

BAYART, Jean-François, 1981. « Le politique par le bas en Afrique noire. Questions de méthode », Politique africaine, 1, p. 53-82. 1993. Religion et modernité politique en Afrique noire. Dieu pour tous et chacun pour soi. Paris : Karthala.

Bayart, Jean-François, Achille Mbembe et Comi M. Toulabor (éds.), 1992. Le politique par le bas : Contribution à une problématique de la démocratie en Afrique noire. Paris : Karthala.

Bernault, Florence et Joseph Tonda, 2000. Politique africaine, 79, « Pouvoirs sorciers » (Dossier).

Bourdieu, Pierre, 1971. « Genèse et structure du champ religieux », Revue française de sociologie, XII, p. 295 -334.

Bratton, Michael, 2003. «Briefing : Islam, Democracy and Public Opinion in Africa », African Affairs, 102, p. 493-501.

Bratton, Michael and Nicholas Van De Walle, 1997. Democratic Experiments in Africa. Cambridge : Cambridge University Press.

Bratton, Michael, Robert Mattes and Emmanuel Gyimah-Boadi (éds.), 2005. Public Opinion, Democracy and Market Reform in Africa. Cambridge : Cambridge University Press.

Brooker, Paul, 2000. Non-Democratic Regimes : Theory, Government and Politics. Houndmills : Macmillan.

CARothers, Thomas, 2002. « The End of the Transition Paradigm », Journal of Democracy, 13 (1), p. 5-21.

Castel, Robert, 1995. Les Métamorphoses de la question sociale. Une chronique du salariat. Paris : Fayard.

Collier, David and Steven Levitsky, 1997. «Research Note : Democracy with Adjectives : Conceptual Innovation in Comparative Research », World Politics, 49 (3), p. 430-451.

Constantin, François et Christian Coulon (éds.), 1997. Religion et transition démocratique en Afrique. Paris : Karthala.

Corten, André et André Mary (éds), 2000. Imaginaires politiques et pentecôtismes. Afrique/Amérique latine. Paris : Karthala.

De Surgy, Albert, 2001. Le phénomène pentecôtiste en Afrique noire : Le cas béninois. Paris : L’Harmattan.

Diamond, Larry Jay, 2001. « Thinking about Hybrid Regimes », Journal of Democracy, 12 (3), p. 21-35.

Diamond, Larry Jay, Martin Lipset and Juan Linz (éds.) 1995. Politics in Developing Countries : Comparing Experiences with Democracy. Boulder, Col. : Lynne Rienner Publishers; London : Adamantine Press.

Dozon, Jean-Pierre, 1995. La cause des prophètes : politique et religion en Afrique contemporaine. Paris : Le Seuil. Eboussi Boulaga, Fabien, 1993. Les conférences nationales en Afrique. Paris : Karthala.

Fourchard, Laurent, André Mary et René OtAyek (dir.), 2005. Entreprises religieuses transnationales en Afrique de l'Ouest. Ibadan et Paris : IFRA et Karthala.

Geschiere, Peter, 1995. Sorcellerie et Politique en Afrique. La viande des autres. Paris : Karthala.

GIFFORD, Paul,

1994. « Some Recent Developments in African Christianity », African Affairs, 93 (373), p. 513-534. 


\section{Entre contestation et légitimation}

1998. African Christianity. Its Public Role. Londres: Hurst.

2004. Ghana's New Christianity : Pentecostalism in a Globalizing African Economy. London, Bloomington, Accra : Hurst, Indiana University Press, Ghana Universities Press.

Haynes, Jeff, 2001. « Transnational Religious Actors and International Politics », Third World Quarterly, 22 (2), p. $143-158$.

Hilgers, Mathieu,

2008. «Politiques urbaines, contestation et décentralisation. Lotissement et représentations sociales au Burkina Faso », Autrepart, 47, p. 209-226.

2009. Une ethnographie à l'échelle de la ville. Urbanité, histoire et reconnaissance à Koudougou. Paris : Karthala.

Hilgers, Mathieu et Jacinthe Mazzocchetti, 2010. Une opposition qui ne dit pas son nom. Représentations et pratiques autour du pouvoir au Burkina Faso. Paris : Karthala.

Holder, Gilles (dir.), 2009. L’Islam, nouvel espace public en Afrique. Paris : Karthala.

Hyden, Goran, 2006. African Politics in Comparative Perspective. Cambridge : Cambridge University Press.

LAurent, Pierre-Joseph,

2003. Les pentecôtistes du Burkina Faso. Mariage, pouvoir et guérison. Paris : IRD-Karthala.

2005. « Le travail missionnaire des assemblées de Dieu du Burkina Faso », in Laurent Fourchard, André Mary et René Otayek (éds.), Entreprises religieuses transnationales en Afrique de l'Ouest, p. 89-110. Ibadan et Paris : IFRA et Karthala.

Levitsky, Steven and Lucan WAy, 2002. «Elections Without Democracy: The Rise of CompetitiveAuthoritarianism», Journal of Democracy, 13 (2), p. 51-66.

Mayrargue, Cédric,

2004. «Trajectoires et enjeux contemporains du pentecôtisme en Afrique de l'Ouest », Critique internationale, 22, p. 95-109.

2008. Les dynamiques paradoxales du pentecôtisme en Afrique subsaharienne. Note de l'IFRI, $21 \mathrm{p}$.

Mbembe, Achille, 1988. Afriques indociles. Paris : Karthala.

McCauley, John F. And Emmanuel Gyimah-Boadi, 2009. « Religious Faith and Democracy : Evidence from the Afrobarometer Surveys », Afrobarometer Working Paper, 113.

Miran, Marie, 2006. Islam, histoire et modernité en Côte d'Ivoire. Paris : Karthala.

Noret, Joël, 2004. « Le pentecôtisme au Togo. Eléments d'histoire et développement », Autrepart, 31, p. 75-92.

Otayeк, René, 1999. Dieu dans la cité : dynamiques religieuses en milieu urbain ouagalais. Bordeaux et Talence : CEAN.

Otayek, René et Benjamin Soares (éds), 2009. Islam, État et société en Afrique. Paris : Karthala.

Otтаway, Marina, 2003. Democracy Challenged : The Rise of Semi-Authoritarianism. Washington DC : Carnegie Endowment for International Peace.

Saul, Mahir et Patrick Royer, 2001. West African Challenge To Empire : Culture and History in the Volta-Bani Anticolonial War. Athens and Oxford : Ohio University Press and James Currey.

Schatzberg, Michael G., 2000. « La sorcellerie comme mode de causalité politique », Politique africaine, 79, p. 333-347.

Schedler, Andreas (éd.), 2006. Electoral Authoritarianism : The Dynamics of Unfree Competition. Boulder : Lynne Rienner Publishers. 


\section{Géraldine André \& Mathieu Hilgers}

Souley, Hassane, Xavier MoYet, Abdourahmane SECK et Maikorema ZAKARI, 2007. Islam, sociétés et politique en Afrique subsaharienne : les exemples du Sénégal, du Niger et du Nigeria. Paris : Les Indes Savantes-Rivages des Xantons.

StrandSBJERg, Camilla,

2000. « Kérékou, God and the Ancestors : Religion and the Conception of Political Power in Benin », African Affairs, 99 (396), p. 395-414.

2005. «Continuité et rupture dans les représentations du pouvoir politique au Bénin entre 1972 et 2001 . Le président Mathieu Kérékou. Du militaire-marxiste au démocrate-pasteur », Cahiers d'études africaines, 177, p. 95-129. 